\title{
Ability of multiaxial fatigue criteria accounting for stress gradient effect for surface defective material
}

\author{
Wichian Niamchaona ${ }^{1, *}$, Fabienne Pennec ${ }^{1}$, Kévin Tihay $^{2}$, Michel Duchet $^{2}$, Bastien Weber $^{2}$, and Jean-Louis Robert $^{1}$ \\ ${ }^{1}$ Institut Pascal, IUT d'Allier, 03100 Montluçon, France \\ ${ }^{2}$ ArcelorMittal, Maizières Research, 57283 Maizières-Les-Metz Cedex, France
}

\begin{abstract}
New high strength steels are widely used nowadays in many industrial areas as in automotive industry. These steels are more resistant and provide higher fatigue limits than latter ones but they are also more sensible to small defects. Natural defects that outcome from metallurgy (as shrinkage, inclusion, void) are not considered in this study. We focus on small manufacturing defects such as cutting edge defects generated by punching or other surface defects due to stamping. These defects are harmful on the material fatigue behaviour due to high stress concentration at defects root. They also generate stress gradient that is beneficial from the fatigue strength point of view. This study focusses on the stress gradient (it does not account for the size effect) from cylindrical defect on specimen edge. Practically a normal stress gradient is added in multiaxial fatigue criteria formulation. Both critical plane approach and integral approach are involved in the present study. This gradient is calculated from stress states at defects root by using FEM. Criteria fatigue function at $N$ cycles is used to assess the material fatigue strength. Obviously multiaxial fatigue criteria accounting for stress gradient give more precise fatigue functions than criteria that do not consider the gradient influence.
\end{abstract}

\section{Introduction}

High strength steels are used in automotive structures such as flask of seat, door bar or suspension arm to reduce their own weight and their energy consumption and to improve their durability. These components are generally made by punching from steel sheets and then by forming through stamping or folding. These processes may generate small defects (figure 1) that can occur on cut edge or external surfaces.
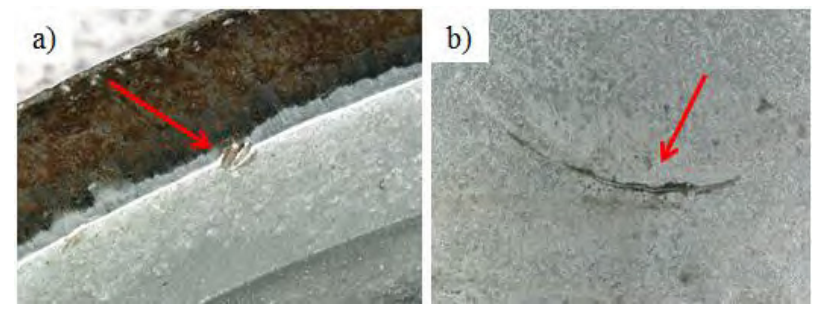

Fig. 1. Small defects occurrence a) on cut edge and b) on external surface of suspension arms.

The small defect may be a cause of fatigue failure in case of variable or constant amplitude loading. Practically it reduces the structure lifetime. It generates some stress concentration that is detrimental from the fatigue point of view. As an evidence, the defect reduces the local fatigue strength but it also generates one stress gradient in the vicinity of the defect. The stress gradient becomes a beneficial phenomenon for the fatigue behaviour of smooth specimens subjected to different fatigue loading types [1]. In the case of tension compression loading no stress gradient occurs as there is one uniform stress field all over the cross section. In the case of rotative or cantilever bending the stress distribution generates some gradient (figure 2) that makes increase the specimen fatigue limit up to $5 \%$ and $10 \%$ respectively.

This study focusses only on the stress gradient induced by the defect without accounting for the size effect of the sample. The stress gradient is integrated in multiaxial fatigue criteria [2] both for critical plane approach [3, 4] and integral approach $[5,6]$. The normal stress gradient is considered as it is much more predominant on fatigue behaviour than shear stress gradient $[7,8,9]$.
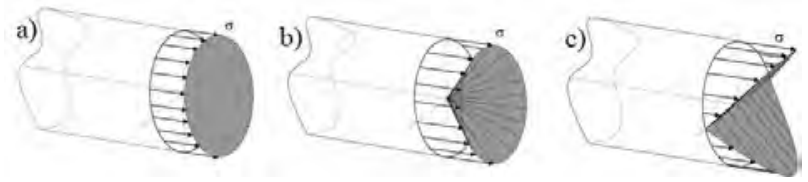

Fig. 2. Stress distribution over the beam cross section for a loading of a) tension-compression b) rotative bending and c) cantilever bending [1].

The stress gradient may be obtained by parabolic interpolation of the stress states over three points (i.e. nodes) at the defect root. The assumption is that fatigue crack initiates at the defect root where some high stress 
concentration occurs. Some researchers $[10,11,12]$ consider the stress gradient to be assessed from one zone which length is equal to $\sqrt{\text { area }}$. The parameter $\sqrt{\text { area }}$ is in fact the square root of the area obtained by the projection of the defect volume over one plane perpendicular to the loading direction [13]. The stress states are obtained for this study by the Femap Nastran Finite Element code.

The outcome of one multiaxial fatigue criterion is a fatigue function $E$ that allows to compare the analysed multiaxial stress cycle to the material fatigue strength at $N$ cycles. In this study the fatigue strength is generally assessed at $2.10^{6}$ cycles, as it is commonly referenced in the automotive industry. The purpose of this paper is mainly to evaluate how accounting for stress gradient gives more precise prediction ability to multiaxial criteria of the actual fatigue strength in the case of surface defect occurrence.

\section{Experimentation}

This section aims to exhibit the experimental fatigue strength reduction that is observed in the case where one surface defect is present. Different defect depths are studied. The experimental fatigue test conditions are described below.

\subsection{Specimen and fatigue tests}

The samples are obtained by cutting from one CP800 high strength steel sheet $2 \mathrm{~mm}$ thick. Sample edges were polished before fatigue testing. Then the cylindrical defect is machined on the edge of the specimen minimal cross section by electro-erosion with diameter wire equal to $200 \mu \mathrm{m}$. The geometry of the defective fatigue specimen is shown in figure 3 .

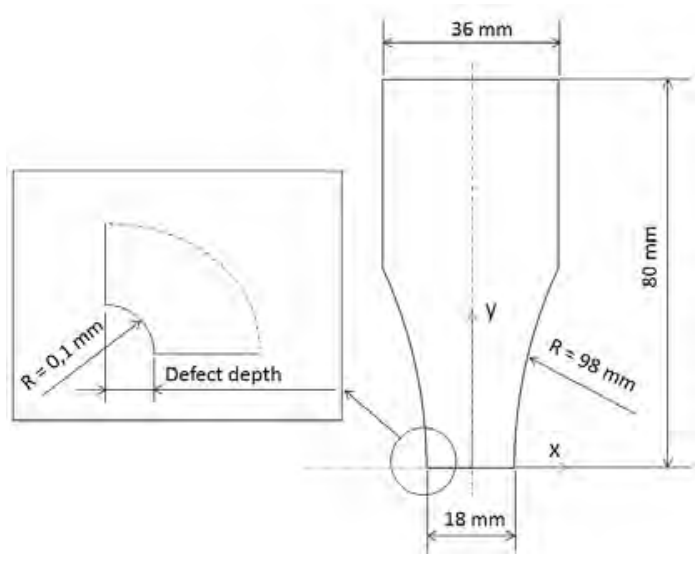

Fig. 3. Geometry of the fatigue specimen with one defect on its edge.

The defect depths vary from $30 \mu \mathrm{m}$ to $300 \mu \mathrm{m}$. The defect radius remains identical for any depth $(\mathrm{R}=0.1$ $\mathrm{mm}$ ). The geometrical sample has a stress concentration factor equal to 1.07 . The $810 \mathrm{MTS}$ fatigue machine is used. Its capacity is a $\pm 100 \mathrm{kN}$ tensile-compressive load.
The test frequency is $20 \mathrm{~Hz}$ and fatigue solicitation is tension with loading ratio equal to 0.1 . The fatigue lifetime threshold is $2.10^{6}$ cycles when no fatigue failure occurs. The defects are observed by a VHX-900F Keyence optical microscope. It allows to measure the real shape and size of the defect after machining. It allows also to observe the fractured section of the sample to identify the crack initiation site and the crack propagation area up to ductile fracture. Other defects shapes and locations have been studied (figure 4). However we focused in this paper on cylindrical defects located on the specimen edge.

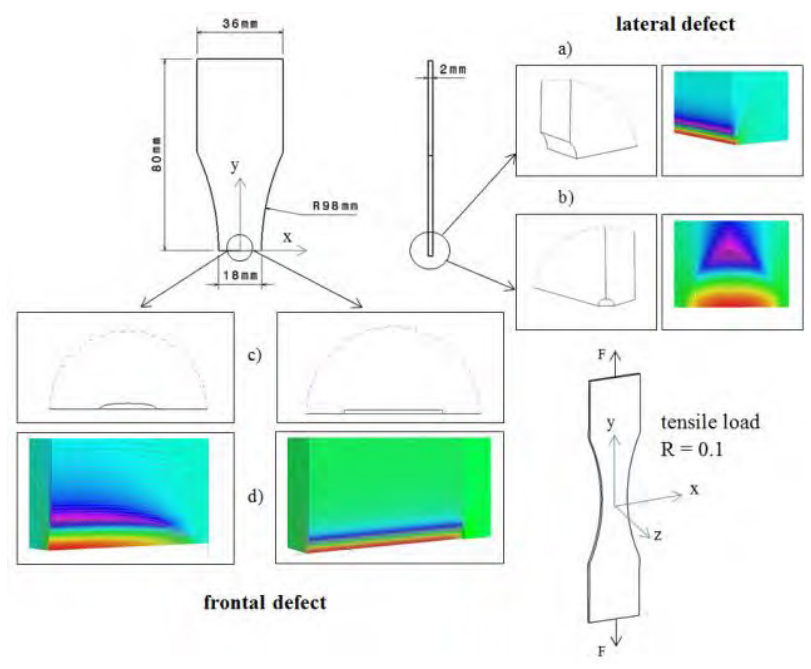

Fig. 4. Geometry and location of studied defects a) on the edge b) in the corner c) and d) on the front surface.

\subsection{Defects fatigue influence}

The results from experiments are shown by S-N curves plotted in figure 5 .

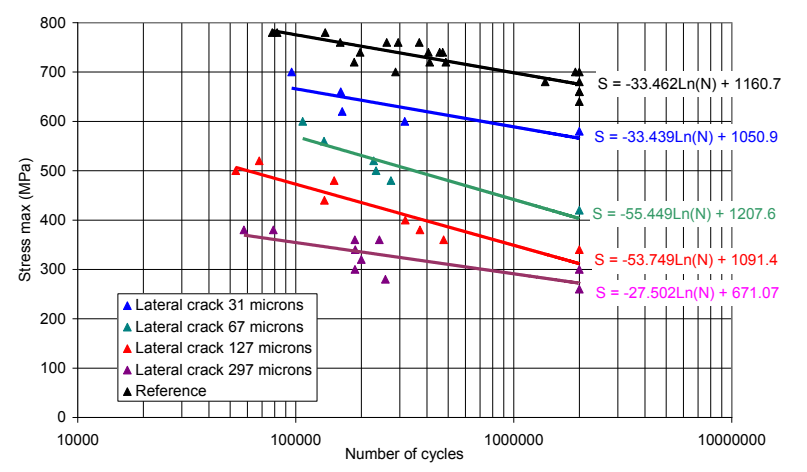

Fig. 5. S-N curves of CP800 steel versus defect depth under tensile load $(\mathrm{R}=0.1)$.

The fatigue strength is reduced when the defect depth increases. The deeper the defect is the most detrimental its influence is from the fatigue resistance point of view. The table 1 gives the experimental fatigue strength up to $2.10^{6}$ cycles for each defect depth.

One has to notice that the defect induced fatigue strengths at $2.10^{6}$ cycles are used as the corresponding 
maximum nominal stress applied to the defected specimen for Finite Element Method calculations.

Table 1. Fatigue strengths at $2.10^{6}$ cycles.

\begin{tabular}{|c|c|}
\hline $\begin{array}{c}\text { Defect depth } \\
(\mu \mathrm{m})\end{array}$ & $\begin{array}{c}\text { Fatigue strength } \\
(\mathrm{MPa})\end{array}$ \\
\hline no defect & 675 \\
\hline 31 & 566 \\
\hline 67 & 403 \\
\hline 127 & 312 \\
\hline 297 & 272 \\
\hline
\end{tabular}

\section{Numerical simulations}

In this section are presented the results of FEM calculation obtained by using Femap Nastran software. Stress fields and stress gradients are determined around defects for fatigue analysis purpose.

\subsection{Stress field near defect root}

The first stress field calculation is realised under a purely elastic stress-strain response assumption. The sample model is meshed with hexahedral elements (QUAD). The mesh size is about $3 \mu \mathrm{m}$ at defect root and then increases afar from defect root in order to reduce calculation time consumption. Symmetry planes are also used so that a quarter of the sample is finally calculated. The tensile load is applied to the specimen along $\mathrm{y}$ direction. The $\sigma_{y y}$ stress field around the defect root is presented in figure 6.a.

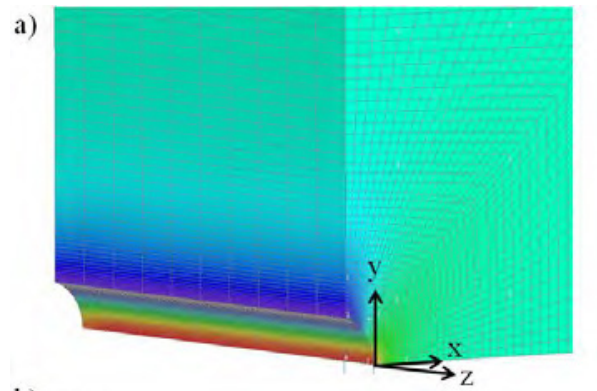

b)

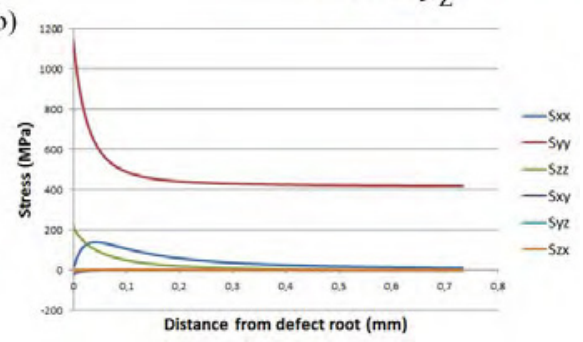

Fig. 6. a) $\sigma_{y y}$ stress concentration at defect root b) elastic response stress components along $\mathrm{x}$-direction at defect root for the sample with a $67 \mu \mathrm{m}$ deep defect.
Stress states are thus multiaxial in the vicinity of the defect (figure 6.b). The stress concentration factor of $\sigma_{y y}$ component is related to the defect depth as summarised in table 2 .

Table 2. Maximum $\sigma_{y y}$ stress value and stress concentration factors.

\begin{tabular}{|c|c|c|c|}
\hline $\begin{array}{c}\text { Defect } \\
\text { depth }(\mu \mathrm{m})\end{array}$ & $\begin{array}{c}\text { Nominal } \\
\text { stress }(\mathrm{MPa})\end{array}$ & $\begin{array}{c}\text { Maximum } \\
\text { elastic stress } \\
(\mathrm{MPa})\end{array}$ & $K_{t}$ \\
\hline $\begin{array}{c}\text { without } \\
\text { defect }\end{array}$ & 675 & 720 & 1.07 \\
\hline 31 & 566 & 1267 & 2.24 \\
\hline 67 & 403 & 1141 & 2.83 \\
\hline 127 & 312 & 1104 & 3.54 \\
\hline 297 & 272 & 1328 & 4.88 \\
\hline
\end{tabular}

Stress states at root defect exhibit triaxiality effect. The highest stress components are $\sigma_{y y}, \sigma_{x x}$ and $\sigma_{z z}$ respectively. Other stress components are close to zero. The stress concentration factor increases with the depth of the defect. The maximum elastic stress at the defect root is beyond the CP800 yield stress $R_{e}$ that is equal to $830 \mathrm{MPa}$. The FEM analysis with elastoplastic stressstrain behaviour is thus the next step. The tensile monotonic elastoplastic stress-strain response for the next numerical calculations is given by figure 7 .

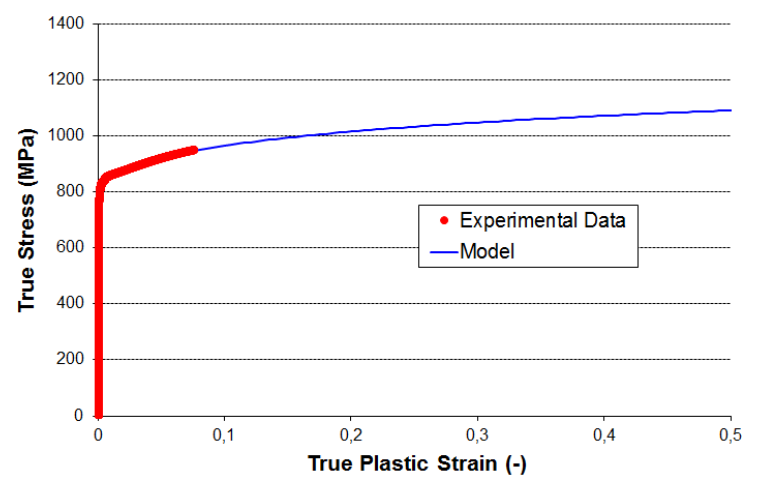

Fig. 7. Tensile monotonic elastoplastic stress-strain response of the CP800 steel.

\subsection{Stress gradient at defect root}

The stress gradient is calculated in the most fatigueprone area of the defective material i.e. at the defect root. The stress gradient is calculated for all the stress components along directions $\mathrm{x}, \mathrm{y}$ and $\mathrm{z}$. The $\mathrm{x}$ direction is normal to the defect root (transverse direction) whereas $\mathrm{z}$ corresponds to the sample thickness direction. One 3-points parabolic interpolation is used to determine all the stress gradients (figure 8). 


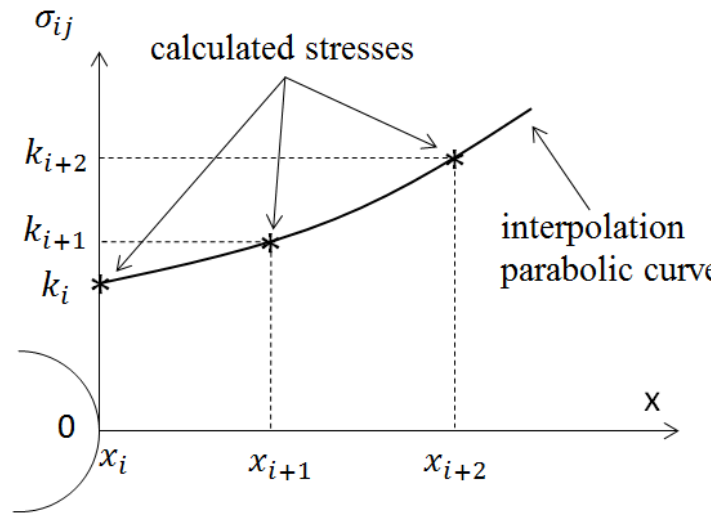

Fig. 8. Parabolic interpolation method used for assessing stress gradients along $\mathrm{x}$ direction at defect root.

The stress component distribution along $\mathrm{x}$ direction is assumed to be modelled by parabolic model, i.e. :

$$
k=a x^{2}+b x+c
$$

Practically the first three nodes at the defect root are retained for the local stress gradient assessment. The actual stress distribution is assumed to check the stress distribution i.e. :

$$
\begin{gathered}
k_{i}=a\left(x_{i}\right)^{2}+b x_{i}+c \\
k_{i+1}=a\left(x_{i+1}\right)^{2}+b x_{i+1}+c \\
k_{i+2}=a\left(x_{i+2}\right)^{2}+b x_{i+2}+c
\end{gathered}
$$

From equation (2), (3) and (4), one obtains :

$$
\begin{gathered}
a=\frac{\left(k_{i+1}-k_{i}\right)\left(x_{i+2}-x_{i}\right)-\left(k_{i+2}-k_{i}\right)\left(x_{i+1}-x_{i}\right)}{\left(x_{i+1}-x_{i}\right)\left(x_{i+2}-x_{i}\right)\left(x_{i+1}-x_{i+2}\right)} \\
b=\frac{\left(k_{i+1}-k_{i}\right)-a\left(x_{i+1}^{2}-x_{i}^{2}\right)}{x_{i+1}-x_{i}}
\end{gathered}
$$

The gradient of one given stress component along direction $\mathrm{x}$ is determined by :

$$
\left(\frac{\partial \sigma}{\partial x}\right)_{x=x_{i}}=\left(\frac{\partial k}{\partial x}\right)_{x=x_{i}}=2 a\left(x_{i}\right)+b
$$

The calculation procedure is carried out for all the stress components of the stress states in the vicinity of the defect root. By this way the following gradient matrix is obtained from the stress gradients of stress components in directions $\mathrm{x}, \mathrm{y}$ and $\mathrm{z}$.

$$
[G]_{\sigma}=\left[\begin{array}{cccccc}
\frac{\partial \sigma_{x x}}{\partial x} & \frac{\partial \sigma_{y y}}{\partial x} & \frac{\partial \sigma_{z z}}{\partial x} & \frac{\partial \sigma_{x y}}{\partial x} & \frac{\partial \sigma_{y z}}{\partial x} & \frac{\partial \sigma_{z x}}{\partial x} \\
\frac{\partial \sigma_{x x}}{\partial y} & \frac{\partial \sigma_{y y}}{\partial y} & \frac{\partial \sigma_{z z}}{\partial y} & \frac{\partial \sigma_{x y}}{\partial y} & \frac{\partial \sigma_{y z}}{\partial y} & \frac{\partial \sigma_{z x}}{\partial y} \\
\frac{\partial \sigma_{x x}}{\partial z} & \frac{\partial \sigma_{y y}}{\partial z} & \frac{\partial \sigma_{z z}}{\partial z} & \frac{\partial \sigma_{x y}}{\partial z} & \frac{\partial \sigma_{y z}}{\partial z} & \frac{\partial \sigma_{z x}}{\partial z}
\end{array}\right]_{x, y, z}
$$

The fatigue influence of the stress gradient is implemented in most multiaxial fatigue criteria through the gradient normal stress $\sigma_{h h}$ that is acting on the material plane which unit normal vector is denoted as $h$. One has :

$$
\sigma_{h h}=[h]^{t} \cdot[\sigma] \cdot[h]
$$

The gradients of this normal stress along axes $\mathrm{x}, \mathrm{y}$ and $\mathrm{z}$ are expressed by equations (10), (11) and (12) respectively.

$$
\begin{aligned}
\frac{\partial \sigma_{h h}}{\partial x}= & h_{x}^{2}\left(\frac{\partial \sigma_{x x}}{\partial x}\right)+h_{y}^{2}\left(\frac{\partial \sigma_{y y}}{\partial x}\right)+h_{z}^{2}\left(\frac{\partial \sigma_{z z}}{\partial x}\right)+ \\
& 2 h_{x} h_{y}\left(\frac{\partial \sigma_{x y}}{\partial x}\right)+2 h_{y} h_{z}\left(\frac{\partial \sigma_{y z}}{\partial x}\right)+2 h_{z} h_{x}\left(\frac{\partial \sigma_{z x}}{\partial x}\right) \\
\frac{\partial \sigma_{h h}}{\partial y}= & h_{x}^{2}\left(\frac{\partial \sigma_{x x}}{\partial y}\right)+h_{y}^{2}\left(\frac{\partial \sigma_{y y}}{\partial y}\right)+h_{z}^{2}\left(\frac{\partial \sigma_{z z}}{\partial y}\right)+ \\
& 2 h_{x} h_{y}\left(\frac{\partial \sigma_{x y}}{\partial y}\right)+2 h_{y} h_{z}\left(\frac{\partial \sigma_{y z}}{\partial y}\right)+2 h_{z} h_{x}\left(\frac{\partial \sigma_{z x}}{\partial y}\right) \\
\frac{\partial \sigma_{h h}}{\partial z}= & h_{x}^{2}\left(\frac{\partial \sigma_{x x}}{\partial z}\right)+h_{y}^{2}\left(\frac{\partial \sigma_{y y}}{\partial z}\right)+h_{z}^{2}\left(\frac{\partial \sigma_{z z}}{\partial z}\right)+ \\
& 2 h_{x} h_{y}\left(\frac{\partial \sigma_{x y}}{\partial z}\right)+2 h_{y} h_{z}\left(\frac{\partial \sigma_{y z}}{\partial z}\right)+2 h_{z} h_{x}\left(\frac{\partial \sigma_{z x}}{\partial z}\right)
\end{aligned}
$$

Finally the gradient of the normal stress along the direction perpendicular to the considered plane is given by equation (13) :

$$
G_{h h}=\frac{\partial \sigma_{h h}}{\partial h}=h_{x}\left(\frac{\partial \sigma_{h h}}{\partial X}\right)+h_{y}\left(\frac{\partial \sigma_{h h}}{\partial Y}\right)+h_{Z}\left(\frac{\partial \sigma_{h h}}{\partial Z}\right)
$$

As a matter of fact multiaxial fatigue criteria take into account this latter gradient from its absolute value $\left|G_{h h}\right|$.

\section{Multiaxial fatigue criteria}

Stress states at defect root are interpreted as the actual multiaxial fatigue strength of CP800 steel up to $N$ cycles by the use of stress gradient dependent multiaxial fatigue criteria. The two most common approaches in multiaxial fatigue, i.e. the critical plane and integral approaches are involved in this study as they are the two main ways for fatigue assessment in multiaxial fatigue. The concept of one multiaxial fatigue criterion is to calculate its own fatigue function that is equal to unity when the analysed multiaxial stress cycle reaches the material fatigue strength up to $N$ cycles. This property may be formulated as :

$E\left\{\left[\sigma_{i j}(t)\right], \sigma_{-1}(N), \tau_{-1}(N), f_{-1}(N), \sigma_{0}(N), \ldots\right\}=1$

where $\left[\sigma_{i j}(t)\right]$ is the analysed multiaxial stress cycle. $\sigma_{-1}(N), \tau_{-1}(N), f_{-1}(N), \sigma_{0}(N), \ldots$ are particular fatigue strengths up to $N$ cycles (for fully reversed tensioncompression load, fully reversed torsion solicitation, fully reversed bending load,...). When the multiaxial stress cycle corresponds to the material fatigue strength up to $N$ cycles, the calculated fatigue function of the criterion can be interpreted as a validity checking of its prediction ability. The $E$ value may be interpreted according to the following manner :

$E<1$ : the criterion is non conservative as it assesses the multiaxial stress cycle under the fatigue strength of the material,

$E>1$ : the criterion is conservative as it states that the multiaxial stress cycle is greater than the material fatigue strength, 
$E=1$ : the criterion is fair as its prediction properly corresponds to the experiment : it agrees the fact that the analysed cycle correspond to material fatigue strength up to $N$ cycles.

\subsection{Conventional fatigue criteria}

This section describes the two employed fatigue criteria. The first one is Dang Van criterion that is based on the so call critical plane approach. This criterion was proposed in 1973 and its formulation uses two stress parameters which are the alternate shear stress $\tau_{h a}(t)$ and hydrostatic pressure $P_{H}(t)$. The damage indicator of the criterion for the material plane which unit normal vector is $h$ is given by :

$$
E_{h}=\max _{t}\left\{\frac{\tau_{h a}(t)+\alpha P_{H}(t)}{\beta}\right\}
$$

The critical plane approach concept can be stated as follows : the so-called critical plane is the most solicited one, with respect to the criterion formulation and drives the material fatigue behaviour. The criterion fatigue function is consequently equal to the criterion damage indicator related to this critical plane. This is written as :

$$
E_{D V}=\max _{h}\left(E_{h}\right)
$$

Two material parameters $\alpha$ and $\beta$ have to be determined from two fatigue limits under different loading types (i.e. tension-compression and torsion) or under two different loading ratio. The determination of the parameters $\alpha$ and $\beta$ is one step that is called the criterion calibration. When the fatigue limits $\left(\sigma_{-1}\right.$ and $\left.\tau_{-1}\right)$ under fully reversed tension-compression and torsion tests $(\mathrm{R}=-1)$ are used, one has :

$$
\alpha=3\left(\frac{\tau_{-1}}{\sigma_{-1}}-\frac{1}{2}\right) \quad \text { and } \quad \beta=\tau_{-1}
$$

When the fatigue strengths $\left(\sigma_{-1}(N)\right.$ and $\left.\tau_{-1}(N)\right)$ under fully reversed tension-compression and torsion tests up to $N$ cycles are used, one states :

$$
\alpha=3\left(\frac{\tau_{-1}(N)}{\sigma_{-1}(N)}-\frac{1}{2}\right) \quad \text { and } \quad \beta=\tau_{-1}(N)
$$

The second fatigue criterion is based on the so-called integral approach. Zenner criterion is used for that purpose. The criterion was proposed in 1993. It defines a fatigue function that is the quadratic mean value of the damage indicator related to all the possible material planes. By this way all these material planes contribute to the fatigue function assessed by the criterion. This concept is clearly distinct from the critical plane approach where only one plane brings its contribution to the fatigue damage assessment. The criterion use four stress parameters : the shear stress amplitude $\tau_{h a}$, the mean shear stress $\tau_{h m}$, the normal stress amplitude $\sigma_{h h a}$ and the mean normal stress $\sigma_{h h m}$. The damage indicator $E_{h}$ related to the $h$ normal vector plane is expressed as :

$$
E_{h}=a \tau_{h a}^{2}\left(1+m \tau_{h m}^{2}\right)+b \sigma_{h h a}^{2}\left(1+n \sigma_{h h m}\right)
$$

The Zenner criterion fatigue function is obtained from :

$$
E_{Z N}=\frac{1}{\sigma_{-1}} \sqrt{\left(\frac{15}{2}\right) \frac{1}{S} \int_{S} E_{h} d S}
$$

The integral concerns all the differently oriented possible material planes by considering a unit radius sphere which area $S$ is equal to $4 \pi$.

Four material parameters $a, b, a m$ and $b n$ have to be determined. The criterion calibration is generally proposed from four fatigue limits : $\sigma_{-1}$ (under fully reversed tension-compression load), $\tau_{-1}$ (under fully reversed torsion load), $\sigma_{0}$ (under repeated tension load) and $\tau_{0}$ (under repeated torsion load) [6]. The expression of the four criterion parameters are given by equation (21) to (24).

$$
\begin{aligned}
& a=\frac{1}{5}\left[3\left(\frac{\sigma_{-1}}{\tau_{-1}}\right)^{2}-4\right] \\
& b=\frac{1}{5}\left[6-2\left(\frac{\sigma_{-1}}{\tau_{-1}}\right)^{2}\right] \\
& a m=\frac{\sigma_{-1}^{2}-\left(\frac{\sigma_{-1}}{\tau_{-1}}\right)^{2}\left(\frac{\tau_{0}}{2}\right)^{2}}{\frac{12}{7}\left(\frac{\tau_{0}}{2}\right)^{4}} \\
& b n=\frac{\sigma_{-1}^{2}-\left(\frac{\sigma_{0}}{2}\right)^{2}-\frac{4}{21} a m\left(\frac{\sigma_{0}}{2}\right)^{4}}{\frac{15}{14}\left(\frac{\sigma_{0}}{2}\right)^{3}}
\end{aligned}
$$

The Dang Van and Zenner criteria formulations presented in this section are stress gradient free. They correspond to the classical or conventional formulation of the criteria as initially proposed by their author. In the next section the stress gradient dependency is introduced in the criteria formulation.

\subsection{Fatigue criteria formulation accounting for stress gradient}

The stress gradient is taken into account by adding a term related to the normal stress gradient to the conventional criteria formulation. This method is inspired from Weber's work in 1999 [1]. This normal stress gradient term introduces a supplementary material parameter $\gamma$ to be determined when the criterion is calibrated. The stress gradient dependent criterion derived from Dang Van formulation is proposed as follows :

$$
E_{h}=\max _{t}\left\{\frac{\tau_{h a}(t)+\alpha P_{H}(t)+\gamma\left|G_{h h}\right|}{\beta}\right\}
$$

The fatigue function of the criterion is still obtained on the critical plane by stating :

$$
E_{D V G}=\max _{h}\left(E_{h}\right)
$$

The material parameters $\alpha$ and $\beta$ are identical to those of the conventional criterion. The parameter $\gamma$ is obtained by the criterion calibration from the fatigue limit obtained for one defective specimen. One has to note 
that the defect size is a reference value. Generally we choose this reference size as the average value of all the fatigue tested defect sizes. The criterion calibration is realized by iterative method as shown in figure 9 .

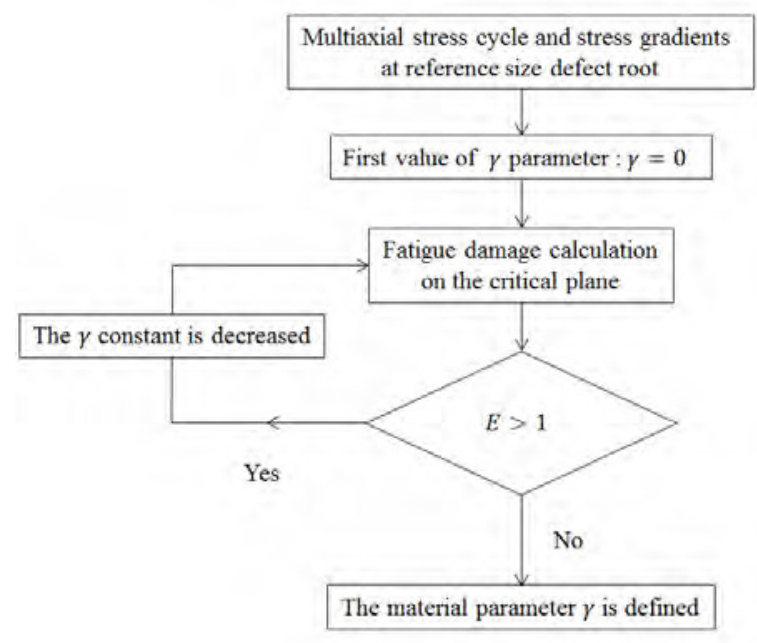

Fig. 9. Iterative method to calibrate the criterion from a defect dependent fatigue test.

For the Zenner criterion the same process is run to integrate within its formulation the stress gradient dependency. Its gradient dependent damage indicator is given by :

$$
E_{h}=a \tau_{h a}^{2}\left(1+m \tau_{h m}^{2}\right)+b \sigma_{h h a}^{2}\left(1+n \sigma_{h h m}\right)+\gamma\left|G_{h h}\right|
$$

The Zenner fatigue function remains analogous. It is expressed by :

$$
E_{Z N G}=\frac{1}{\sigma_{-1}} \sqrt{\left(\frac{15}{2}\right) \frac{1}{S} \int_{S} E_{h} d S}
$$

The material parameters $a, b, a m$ and $b n$ are still the same as for conventional formulation of the criterion. The material parameter $\gamma$ is determined by the calibration of the criterion on one fatigue strength obtained for specimen with an average size defect. The same iterative method than for Dang Van criterion is employed to determine $\gamma$.

\subsection{Validation of the gradient dependent criteria}

The fatigue functions of the Dang Van and Zenner modified criteria are calculated for fatigue strengths at $2.10^{6}$ cycles. They are compared with those obtained with the conventional multiaxial criteria. The fatigue strengths were obtained from $\mathrm{R}=0.1$ uniaxial tension fatigue tests. The Haigh's diagram according to Gerber parabolic model is then used to assess the fatigue strength of CP800 steel under fully reversed $(\mathrm{R}=-1)$ and repeated $(\mathrm{R}=0)$ tension. The two torsion fatigue strengths under fully reversed load and repeated load are obtained from :

$$
\tau_{-1}=\frac{\sigma_{-1}}{\sqrt{3}}
$$

$$
\tau_{0}=\frac{4 \sigma_{-1}}{\frac{2 \sigma_{-1}}{\sigma_{0}}+1}
$$

The fatigue strength for defective material that is used for the criteria calibration has a $127 \mu \mathrm{m}$ deep defect (that is the average depth of fatigue tested defective specimen). The fatigue functions are given in table 3 .

Table 3. Fatigue functions assessment from conventional and gradient dependent criteria.

\begin{tabular}{|c|c|c|c|c|}
\hline \multirow{2}{*}{$\begin{array}{c}\text { Defect } \\
\text { depth }(\mu \mathrm{m})\end{array}$} & \multicolumn{4}{|c|}{ Fatigue function at $2.10^{6}$ cycles } \\
\cline { 2 - 5 } & $\begin{array}{c}\text { Dang } \\
\text { Van }\end{array}$ & DVG & Zenner & ZNG \\
\hline 31 & 1.49 & 0.97 & 1.41 & 0.98 \\
\hline 67 & 1.47 & 0.96 & 1.40 & 0.95 \\
\hline 127 & 1.47 & 1.00 & 1.40 & 1.00 \\
\hline 297 & 1.54 & 1.05 & 1.45 & 1.08 \\
\hline
\end{tabular}

The fatigue functions assessment obtained for the different experimentally tested defect depths are plotted on figure 10. Criteria integrating the stress gradient effect give a strongly better accuracy of the CP800 steel fatigue strength when the specimens contain defects whatever the size. Conventional fatigue criteria strongly underestimate the real fatigue strength of the material with cylindrical defects located on the specimen edge.

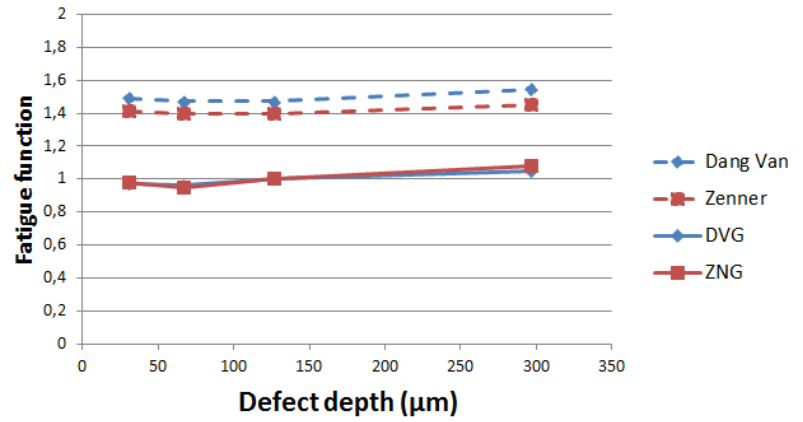

Fig. 10. Comparison of fatigue predictions between conventional and stress gradient dependent fatigue criteria against fatigue test results of defective specimen.

\section{Conclusion}

The CP800 high strength steel that is mainly devoted to automotive application has been studied against fatigue with the presence of surface defect. The defect reduces the fatigue strength of the steel because of the local stress concentration factor that exists in the vicinity of the defect root. Moreover this fatigue strength decrease cannot be properly predicted without accounting for the stress gradient induced in the highly stressed material volume.

The purpose of the presented work is to assess how multiaxial fatigue criteria are able to predict the steel 
fatigue behaviour is such cases of high stress gradient. Two multiaxial fatigue approaches, the critical plane and the integral concepts, are investigated to account for stress gradient and to predict the fatigue strength of the defective steel. Dang Van and Zenner criteria are the most commonly used multiaxial stress criteria and have been modified in order to integrate the stress gradient effect in their formulation. The ability of these two criteria is scanned over the whole range of experimental fatigue test results, with defect depth from $30 \mu \mathrm{m}$ up to $300 \mu \mathrm{m}$. Prediction obtained for gradient dependent criteria are compared to those realized with the conventional criteria.

A quite good precision is obtained for the gradient dependent criteria formulation whereas conventional criteria underestimate the material fatigue strength with an amount about $40 \%$. An other peculiarity concerns the stress gradients that are observed around the defect root. They are really higher than stress gradients that can be observed on smooth specimen for particular loading as bending or torsion solicitation. The ability of multiaxial criteria to correctly depict the stress gradient influence on the material fatigue behaviour around defect requires to make the criterion calibration from one fatigue test realized on a defective specimen.

\section{References}

1. B. Weber, Fatigue multiaxiale des structures industrielles sous chargement quelconque, Thesis INSA Lyon, (1999)

2. B. Weber, B. Kenmeugne, J.C. Clement, J.L. Robert, Comp. Mat. Sci., 15 (1999)

3. K. Dang Van, A. Le Douaron, H.P. Lieurade, Advances in Fracture Research, (New Dehli, 1984)

4. K. Dang Van, B. Griveau, O. Message, Mec. E. Pub., (1989)

5. J. Liu, H. Zenner, Mat.-wiss. u. Werksofftech, 24 (1993)

6. H. Zenner, A. Simbürger, J. Liu, Int. J. Fat., 22 (2000)

7. I.V. Papadopolos, V.P Panoskaltsis, E. Frac. Mec., 55, 4 (1996)

8. D.H. Luu, M.H. Maitournam, Q.S Nguyen, Int. J. Fat., 61 (2014)

9. M. Zepeng, P. Le Tallec, H. Maitournam, Proc. E., 133 (2015)

10. T. Billaudeau, Fatigue multiaxiale des matériaux à défauts: mécanismes et critère d'endurance, Thesis ENSMA Poitiers, (2002)

11. Y. Nadot, T. Billaudeau, E. Frac.Mec., 73 (2006)

12. A. Nadot, P. Mu, C. Nadot-Martin, A. Steinwandel, The Tenth International Conference on Multiaxial Fatigue \& Fracture, (ICMFF10, Kyoto, 2013)

13. Y. Murakami, Metal fatigue : Effect of small defects and nonmetallic inclusions, (2002) 